\title{
SPECTRAL EXPANSION OF THE ROVIBRATIONAL ENERGY OF DIATOMIC MOLECULES DESCRIBED BY MORSE POTENTIAL*
}

\author{
M. MoLSKI \\ Department of Theoretical Chemistry, Faculty of Chemistry \\ Adam Mickiewicz University \\ Grunwaldzka 6, 60-780 Poznań, Poland \\ (Received June 3, 1993; revised version July 26, 1993)
}

\begin{abstract}
On the basis of the deformable body model and the Morse potential approximation a six-parametric spectral expansion of the rovibrational energy of diatomic molecules, competitive to the standard Dunham approach, is proposed. The considered formula takes into account the centrifugal distortion effect and anharmonic corrections, which permit investigation of the molecular spectra over a wide range of rotational and vibrational states.
\end{abstract}

PACS numbers: $33.10 . \mathrm{Cs}, 33.10 . \mathrm{Jz}$

\section{Introduction}

A simple analysis of rovibrational spectra of diatomic molecules is based on the Schrödinger equation

$$
\left[-\frac{\hbar^{2}}{2 m} \frac{\partial^{2}}{\partial r^{2}}+\frac{\hbar^{2} J(J+1)}{2 m r^{2}}+U(r)-E_{v J}\right] \psi_{v J}(r)=0,
$$

in which $r$ denotes the internuclear separation, $m$ is the reduced mass of a diatomic system and $U(r)$ is the internuclear potential containing a set of parameters to be obtained by a fit to the experimental energy levels or, if available, by a fit to a theoretically calculated potential. One of the methods permitting determination of the energy eigenvalues $E_{v J}$ for Eq. (1) is the Dunham approach [1] in which the potential is assumed to be a power series of the instantaneous reduced displacement from the equilibrium $x=\left(r-r_{0}\right) / r_{0}$

$$
U(r)=a_{0} x^{2}\left(1+\sum_{n=1} a_{n} x^{n}\right) .
$$

\footnotetext{
*This research was supported by the Committee for Scientific Research grant 206639101.
} 
Application of the WKB method to Eq. (1) including potential (2) leads directly to the eigenvalues taking the form of the series of vibration and rotation quantum numbers

$$
E_{v J}=\sum_{l, m} Y_{l m}(v+1 / 2)^{l} J^{m}(J+1)^{m}
$$

where $Y_{l m}$ are given as functions of the potential parameters $\left\{a_{n}\right\}$ and the equilibrium distance $r_{0}$. The Dunham expansion has been tested on many molecules providing quite satisfactory relationships between theoretical and experimental values of rovibrational energy [2], and giving useful information on internal motion potential $[3,4]$. However, this method has three main disadvantages, namely:

(i) The Dunham function (2) including the potential parameters $\left\{a_{n}\right\}$ obtained by the fit to experimental data, frequently differs from RKR curves which are commonly regarded as true molecular potential curves.

(ii) The Dunham expansion (3) has a poor convergence, and sometimes it is necessary to use up to a dozen parameters $\left\{a_{n}\right\}$ to obtain a good reproduction of the transitions observed.

(iii) The Dunham approach cannot provide any information about the wave functions of individual states of a molecule. Therefore, the matrix elements of various quantum-mechanical operators, the Franck-Condon factors and transitions intensities, cannot be directly calculated.

In order to overcome the above difficulties a new expansion of the rovibrational energy of diatomic molecules, based on the deformable body model [5] and harmonic potential approximation, has been proposed $[6,7]$. The obtained formula preserves the analytic form of energy eigenvalues and corresponding wave functions, and satisfactorily reproduces the molecular parameters and rovibrational spectra over a wide range of rotational states. However, the harmonic approximation does not permit an investigation of the overtone transitions and limits the application of the formula obtained to the molecules with small deformational susceptibility and low excited vibrational states.

Recently, on the basis of the deformable body model and the Morse potential approximation [8], an energy formula describing the rovibrational states of diatomic molecules has been derived [9], which takes into account the centrifugal distortion effect and has the form of a two-element continued fraction similar to that obtained for harmonic approximation [7]. This paper is an attempt at treating the above mentioned equation as the 6-parametric expansion of the rovibrational energy, and employing it for straightforward evaluation of the spectral parameters $\{\omega, \omega x, \alpha, B, C, D\}$ as well as for assignment of the rovibrational spectra of the selected diatomic molecules. For our purpose, the $\mathrm{HF},{ }^{132} \mathrm{XeH}^{+}$, and ${ }^{7} \mathrm{LiH}$ will be taken as test molecules.

\section{Method and applications}

In our previous work [9] it has been shown that energy eigenvalues of the rovibrational Schrödinger equation (1) including the simple 3-parametric Morse potential [8], can be given in the form 


$$
\begin{aligned}
& E_{v J}=\omega(J)(v+1 / 2)-\omega x(v+1 / 2)^{2}-\alpha(J)(v+1 / 2) J(J+1) \\
& \quad+B(J) J(J+1)-D(J) J^{2}(J+1)^{2}, \\
& \omega(J)=\omega[1+C J(J+1)]^{-1 / 2}, \quad \alpha(J)=\alpha[1+C J(J+1)]^{-1 / 2}, \\
& B(J)=B[1+C J(J+1)]^{-1}, \quad D(J)=D[1+C J(J+1)]^{-1},
\end{aligned}
$$

where the spectral parameters $\{\omega, \omega x, \alpha, B, C, D\}$ can be expressed by the potential ones as follows:

$$
\begin{aligned}
& B=\hbar^{2}\left(2 m r_{0}^{2}\right)^{-1}, \quad \omega=2 a \sqrt{B D_{0}}, \quad \omega x=a^{2} B, \\
& C=B D_{0}^{-1}\left(-a^{-1}+3 a^{-2}\right), \quad D=B^{2} D_{0}^{-1}\left(a^{-1}-2 a^{-2}\right), \\
& \alpha=B^{3 / 2} D_{0}^{-1 / 2} a\left(4 a^{-1}-6 a^{-2}\right) .
\end{aligned}
$$

In the above equations $r_{0}$ denotes the equilibrium distance, $D_{0}$ represents the depth of the potential well (related to the dissociation energy of a molecule), whereas $a$ is the range potential parameter.

The wave functions corresponding to the eigenvalues (4) can be given as follows [9]:

$$
\begin{aligned}
& \psi_{v J}=y^{\eta_{v J}} \exp (-y / 2)_{1} F_{1}(b, c ; y), \\
& y=2 a_{J}^{-1} \sqrt{D_{J} B_{J}^{-1}} \exp \left[-a_{J}\left(r-r_{J}\right) / r_{J}\right], \quad \eta_{v J}=\sqrt{E_{v J} B_{J}^{-1}} \\
& c=2 \eta_{v J}+1, \quad b=c / 2-a^{-1} \sqrt{D_{J} B_{J}}, \\
& r_{J}=r_{0}\left\{1-a^{-1} \ln \left[\frac{D_{0}-\left(4 a^{-1}-6 a^{-2}\right) B J(J+1) / 2}{D_{0}+\left(-a^{-1}+6 a^{-2}\right) B J(J+1)}\right]\right\} \\
& a_{J}=a\left\{1-a^{-1} \ln \left[\frac{D_{0}-\left(4 a^{-1}-6 a^{-2}\right) B J(J+1) / 2}{D_{0}+\left(-a^{-1}+6 a^{-2}\right) B J(J+1)}\right]\right\} \\
& B_{J}=\hbar^{2}\left(2 m r_{J}^{2}\right)^{-1} \\
& D_{J}=r_{0}^{2} a^{-2}\left[D_{0}+\left(-a^{-1}+3 a^{-2}\right) B J(J+1)\right] \exp \left[-2 a\left(r_{J}-r_{0}\right) / r_{0}\right],
\end{aligned}
$$

where ${ }_{1} F_{1}(b, c ; y)$ denotes the confluent hypergeometric (or Kummer) function.

The obtained eigenvalues (4) consist of the terms which have clear physical interpretation. Namely, the first two ones represent the energy of a harmonic oscillator with a rotational dependence of the fundamental frequency $\omega$, and the anharmonic energy'not perturbed by rotation, the third describes a coupling between rotation and vibration including a rotational dependence of the constant $\alpha$, whereas the last two terms are the effective rotation energy in which a modification of the molecular constants $B$ and $D$, in the $J$-rotational state is taken into account. A detailed analysis of (4) leads to a few interesting conclusions:

(i) For a small value of the constant $C$, the terms describing the effective rotation and vibration energy can be expanded in a power series of the rotational quantum number, leading to the polynomial Dunham expansion.

(ii) The eigenvalues (4) take the form of a two-element continued fraction [10] which seems to be a more general equation describing rovibrational states of diatomic molecules than the polynomial expansion. 
(iii) The inclusion of the anharmonic term and rotational dependence of the $\omega, \alpha, B$, and $D$ constants permits introduction of the high-order corrections to the rovibrational energy, and investigation of the molecular spectra over a wide range of rotational and vibrational states.

(iv) The considered formula is a more strongly physically supported equation describing rovibrational states of diatomic molecules than that derived on the basis of the harmonic potential $[6,7]$.

In the light of the above facts, the formula (4) may be considered as a 6-parametric spectral expansion of the rovibrational energy of diatomic molecules, competitive to the standard 6-parametric Dunham formula [1]. Consequently, it may be employed for straightforward evaluation of the spectral parameters $\{\omega, \omega x, \alpha, B, C, D\}$ (formally treated as independent parameters), and then for prediction of the rovibrational spectra of diatomic molecules.

TABLE I

Energy of rovibrational transitions

$\Delta E=E_{\text {obs }}^{a}-E_{\text {calc }}\left[\mathrm{cm}^{-1}\right]$ for $R(J)$ branch, $v=0 \rightarrow 1,0 \rightarrow 2$, of $\mathrm{HF}$ molecule.

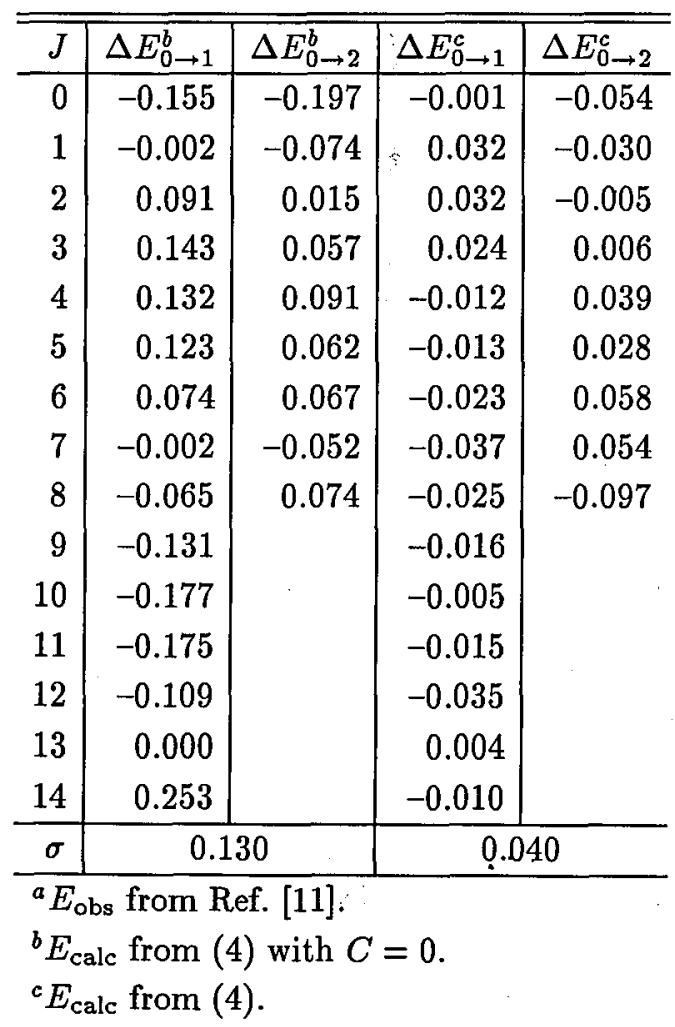




\section{TABLE II}

Energy of rovibrational transitions $\Delta E=$ $E_{\text {obs }}^{a}-E_{\text {calc }}\left[\mathrm{cm}^{-1}\right]$ for $R(J)$ branch, $v=$ $0 \rightarrow 1,1 \rightarrow 2$, of ${ }^{132} \mathrm{XeH}^{+}$molecule.

\begin{tabular}{|c|c|c|c|c|}
\hline$J$ & $\Delta E_{0 \rightarrow 1}^{b}$ & $\Delta E_{1 \rightarrow 2}^{b}$ & $\Delta E_{0 \rightarrow 1}^{c}$ & $\Delta E_{1 \rightarrow 2}^{c}$ \\
\hline 0 & -0.0330 & -0.0399 & -0.0141 & 0.0018 \\
\hline 1 & -0.0059 & -0.0179 & -0.0065 & 0.0057 \\
\hline 2 & 0.0216 & -0.0010 & 0.0055 & 0.0081 \\
\hline 3 & 0.0328 & 0.0079 & 0.0059 & 0.0063 \\
\hline 4 & 0.0398 & 0.0083 & 0.0073 & 0.0003 \\
\hline 5 & 0.0360 & 0.0070 & 0.0032 & -0.0033 \\
\hline 6 & 0.0291 & -0.0008 & 0.0010 & -0.0099 \\
\hline 7 & 0.0185 & & -0.0004 & \\
\hline 8 & 0.0030 & -0.0096 & -0.0033 & -0.0092 \\
\hline 9 & -0.0182 & -0.0205 & -0.0034 & -0.0146 \\
\hline 10 & -0.0239 & -0.0174 & -0.0003 & -0.0080 \\
\hline 11 & -0.0344 & -0.0067 & 0.0025 & 0.0018 \\
\hline 12 & -0.0356 & -0.0014 & 0.0101 & -0.0011 \\
\hline 13 & 0.0373 & 0.0286 & 0.0098 & 0.0102 \\
\hline 14 & -0.0140 & 0.0634 & -0.0012 & 0.0121 \\
\hline 15 & 0.0152 & & -0.0160 & \\
\hline$\sigma$ & \multicolumn{2}{|c|}{0.0279} & \multicolumn{2}{|c|}{0.0085} \\
\hline \multicolumn{5}{|c|}{${ }^{a} E_{\text {obs }}$ from Ref. [12]. } \\
\hline
\end{tabular}

this paper we propose to perform the suitable calculations for $\mathrm{HF},{ }^{132} \mathrm{XeH}^{+}$ and ${ }^{7} \mathrm{LiH}$ molecules. The best values of the spectral parameters will be determined by the least squares routine in which the statistical weights, proportional to the inverse of experimental uncertainties, are taken as equal to one. The uncertainty in parentheses is one standard deviation in units of the last quoted digit of the fitted parameters. The rovibrational transitions are calculated from (i) the 5-parametric Dunham expansion (4) obtained for $C=0$, and (ii) the full 6-parametric formula (4). The calculated frequencies and the sigma standard errors of the fit are presented in Tables I-III, whereas Tables IV and V report the spectral parameters obtained by the fitting procedure, and the potential parameters $\left\{r_{0}, D_{0}, a\right\}$ calculated on the basis of Eqs. (7)-(9).

Obviously, from the mathematical point of view, the spectral parameters are not independent of each other, therefore $\left\{r_{0}, D_{0}, a\right\}$ can be determined in a few different ways. To this purpose we propose to employ the set $\{\omega, \omega x, B\}$, in the 
TABLE III

Energy of rovibrational transitions $\Delta E=$ $E_{\text {obs }}^{a}-E_{\text {calc }}\left[\mathrm{cm}^{-1}\right]$ for $R(J)$ and $P(J)$ branches, $v=0 \rightarrow 1$, of ${ }^{7} \mathrm{LiH}$ molecule.

\begin{tabular}{|c|c|c|c|c|}
\hline$J$ & $\Delta E_{R}^{b}$ & $\Delta E_{P}^{b}$ & $\Delta E_{R}^{c}$ & $\Delta E_{P}^{c}$ \\
\hline 0 & 0.0450 & & -0.0006 & \\
\hline 1 & 0.0540 & 0.0184 & 0.0003 & -0.0007 \\
\hline 2 & 0.0574 & 0.0024 & 0.0002 & -0.0008 \\
\hline 3 & 0.0560 & -0.0139 & 0.0006 & -0.0010 \\
\hline 4 & 0.0484 & -0.0285 & 0.0003 & -0.0006 \\
\hline 5 & 0.0360 & -0.0407 & 0.0004 & -0.0006 \\
\hline 6 & 0.0196 & -0.0491 & 0.0010 & -0.0002 \\
\hline 7 & -0.0010 & -0.0529 & 0.0006 & 0.0001 \\
\hline 8 & -0.0228 & -0.0515 & 0.0004 & 0.0005 \\
\hline 9 & -0.0430 & -0.0448 & 0.0004 & 0.0008 \\
\hline 10 & -0.0594 & -0.0332 & -0.0003 & 0.0008 \\
\hline 11 & -0.0667 & -0.0170 & -0.0007 & 0.0008 \\
\hline 12 & -0.0604 & 0.0025 & -0.0009 & 0.0009 \\
\hline 13 & -0.0352 & 0.0230 & -0.0014 & 0.0005 \\
\hline 14 & 0.0176 & 0.0422 & -0.0001 & 0.0000 \\
\hline 15 & 0.1040 & 0.0573 & 0.0015 & -0.0004 \\
\hline 16 & & 0.0638 & & -0.0009 \\
\hline 17 & & 0.0576 & & -0.0009 \\
\hline 18 & & 0.0325 & & -0.0008 \\
\hline 19 & & -0.0180 & & -0.0006 \\
\hline 20 & & -0.0995 & & 0.0016 \\
\hline$\sigma$ & \multicolumn{2}{|c|}{0.0509} & \multicolumn{2}{|c|}{0.0008} \\
\hline
\end{tabular}

case of $\mathrm{HF}$ and ${ }^{132} \mathrm{XeH}^{+}$molecules, which provides the following relations:

$$
r_{0}=\sqrt{\hbar^{2} / 2 m B}, \quad D_{0}=\omega^{2} / 4 \omega x, \quad a=\sqrt{\omega x / B},
$$

whereas for ${ }^{7} \mathrm{LiH}$ we take $\{\omega, \alpha, B\}$, and the potential parameters will be determined from the relations

$$
\begin{aligned}
& r_{0}=\sqrt{\hbar^{2} / 2 m B}, \quad a=\alpha \omega\left(8 B^{2}\right)^{-1}+3 / 2 \\
& D_{0}=\omega^{2}\left\{4\left[\alpha \omega /\left(8 B^{2}\right)+3 / 2\right]^{2} B\right\} .
\end{aligned}
$$


TABLE IV

Ground state molecular constants $\left[\mathrm{cm}^{-1}\right]$ for $\mathrm{HF},{ }^{132} \mathrm{XeH}^{+}$and ${ }^{7} \mathrm{LiH}$ molecules, fitted to the $N^{2}$ experimental frequencies.

\begin{tabular}{l|l|l|l|l|l|}
\hline \hline \multicolumn{1}{c|}{$\omega$} & \multicolumn{1}{c|}{$\omega x$} & \multicolumn{1}{c|}{$\alpha$} & \multicolumn{1}{c|}{$B$} & \multicolumn{1}{c}{$D \times 10^{4}$} & \multicolumn{1}{c}{$C \times 10^{4}$} \\
\hline \multicolumn{6}{c}{ HF } \\
\hline $4134.09(29)$ & $86.167(92)$ & $0.7499(18)$ & $20.820(16)$ & $20.17(20)$ & \\
$4133.48(10)$ & $86.008(31)$ & $0.430(21)$ & $20.8999(78)$ & $-7.9(18)$ & $1.58(10)$ \\
$4138.32^{b}$ & $89.88^{b}$ & $0.798^{b}$ & $20.9557^{b}$ & $21.51^{b}$ & \\
\hline \multicolumn{6}{c}{$132 \mathrm{XeH}^{+}$} \\
\hline $2270.058(40)$ & $41.3243(89)$ & $0.1786(84)$ & $6.5266(48)$ & $2.741(77)$ & \\
$2270.038(12)$ & $41.3349(28)$ & $0.0782(53)$ & $6.5392(17)$ & $-2.39(27)$ & $0.891(47)$ \\
$2269.9674^{c}$ & $41.32056^{c}$ & $0.186686^{c}$ & $6.560686^{c}$ & $2.1907^{c}$ & \\
\hline \multicolumn{7}{c}{${ }^{7} \mathrm{LiH}$} \\
\hline $1359.675(16)$ & $9.52(96)$ & $0.21111(28)$ & $7.5041(10)$ & $8.012(24)$ & \\
$1359.70885(28)$ & $4.28(36)$ & $0.12944(24)$ & $7.512196(29)$ & $-0.0597(25)$ & $1.2212(35)$ \\
$1405.65^{b}$ & $23.20^{b}$ & $0.2132^{b}$ & $7.5131^{b}$ & $8.617^{b}$ & \\
\hline
\end{tabular}

${ }^{a}$ For HF $N=24,{ }^{132} \mathrm{XeH}^{+} N=30$, and for ${ }^{7} \mathrm{LiH} N=36$.

${ }^{b}$ Data from Ref. [14].

c Data from Ref. [12].

TABLE V

The Morse potential parameters for molecules considered.

\begin{tabular}{l|l|c|c|c}
\hline \hline & \multicolumn{1}{|c|}{$r_{\exp }[\AA]$} & $r_{0}[\AA]$ & $D_{0}\left[\mathrm{~cm}^{-1}\right]$ & $a^{a}$ \\
\hline $\mathrm{HF}$ & $0.916808^{b}$ & 0.918029 & 49662.934 & 2.028603 \\
${ }^{132} \mathrm{XeH}^{+}$ & $1.60281(6)^{c}$ & 1.605448 & 31166.602 & 2.514182 \\
${ }^{7} \mathrm{LiH}$ & $1.5957^{b}$ & 1.595760 & 17227.138 & 1.889844 \\
\hline
\end{tabular}

a Parameter dimensionless.

${ }^{b}$ Data from Ref. [14].

${ }^{c}$ Data from Ref. [12].

The reason for this choice is that among the first- and second-order spectral parameters only these have the relatively smallest standard errors (see Table IV), ensuring high precision in evaluation of the potential parameters.

\section{Discussion}

The performed calculations indicate that the proposed spectral expansion of the rovibrational energy of diatomic systems reproduces quite satisfactorily rovibrational transitions and spectral parameters of the molecules considered in a wide range of rotational and vibrational states. Inspection of Tables I-II reveals that introduction of the $C$-parameter generating fraction continued terms, improves the accuracy of calculations by approximately one $\left(\mathrm{HF},{ }^{132} \mathrm{XeH}^{+}\right)$, and two $\left({ }^{7} \mathrm{LiH}\right)$ orders of magnitude, respectively. As the Morse potential allows for the overtone transitions, the obtained formula may be applied in investigation of vibrational transitions in a greater range of the quantum number of vibrations than the formula obtained on the basis of the harmonic potential $[6,7]$. Application of the 
Morse function permits also to include the anharmonic corrections to rovibrational energy, making it possible to investigate the molecules with an anharmonic type of vibrational motion.

Needless to mention that the presented approach provides the rovibrational wave function (10), including the molecular parameters $\left\{r_{0}, D_{0}, a\right\}$ calculated from the spectral ones. In this way the main disadvantage of the Dunham approach is overcome.

The proposed expansion may be of limited use in advanced rovibrational spectroscopy of diatomic systems, because of a low number of fitted parameters, however, in the case of molecules of poor spectra (a small number of observed transitions) it is quite sufficient for a good reproduction of the molecular spectra. Moreover, the fact that the presented approach besides the 6-parametric spectral expansion provides also the corresponding wave functions in the analytical form, favours our method to that of Dunham, as permitting a more sophisticated analysis of rovibrational spectra.

\section{Acknowledgment}

I wish to thank Professor J. Konarski for stimulating and constructive discussions.

\section{References}

[1] J.L. Dunham, Phys. Rev. 41, 713 (1932).

[2] I.M. Mills, Specialist Periodical Report-Theoretical Chemistry, Vol. 1, Eds. R.N. Dixon, C. Thompson, American Chemical Society, Washington DC 1974, p. 110.

[3] R.N. Zarea, A.L. Schmeltekopf, W.J. Harrod, D.L. Albritton, J. Mol. Spectrosc. 46, 37 (1973).

[4] D.L. Albritton, W.J. Harrod, A.L. Schmeltekopf, R.N. Zarea, J. Mol. Spectrosc. 46, 67 (1973).

[5] M. Molski, Acta Phys. Pol. A 76, 877 (1989).

[6] M. Molski, Acta Phys. Pol. A 81, 485 (1992).

[7] M. Molski, J. Mol. Struct. 275, 13 (1992).

[8] P.M. Morse, Phys. Rev. 34, 57 (1927).

[9] M. Molski, Acta Phys. Pol. A 83, 417 (1993).

[10] W.E . Naniłow, A.N. Iwanowa, J.K. Isakowa, L.A. Lusternik, G.S. Salechow, A.N. Chowanski, L.J. Cłaf, A.R. Janpolski, Funkcje, granice, szeregi, ułamki łańcuchowe, PWN, Warszawa 1970, p. 248.

[11] D.U. Webb, K. Narahari Rao, J. Mol. Spectrosc. 28, 121 (1968).

[12] S.A. Rogers, C.R. Brazier, P.F. Bernath, J. Chem. Phys. 87, 157 (1987).

[13] A.G. Maki, W.B. Olson, G. Thompson, J. Mol. Spectrosc. 144, 257 (1990).

[14] K.P. Huber, G. Herzberg, Constants of Diatomic Molecules, Van Nostrand Reinhold, New York 1979. 